\title{
Microscopic Scenario for Striped Superconductors
}

\author{
J. Eroles ${ }^{\text {a b }}$, G. Ortiz ${ }^{a}$, A.V. Balatsky ${ }^{a}$, and A.R. Bishop ${ }^{a}$

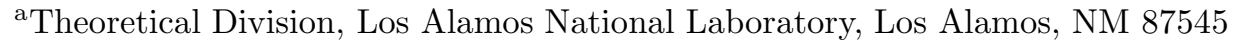 \\ ${ }^{\mathrm{b}}$ Centro Atómico Bariloche and Instituto Balseiro, S. C. de Bariloche, Argentina (8400)
}

We argue that the superconducting state found in high- $T_{c}$ cuprates is inhomogeneous with a corresponding inhomogeneous superfluid density. We introduce two classes of microscopic models which capture the magnetic and superconducting properties of these strongly correlated materials. We start from a generalized $t-J$ model, in which appropriate inhomogeneous terms mimic stripes. We find that inhomogeneous interactions that break magnetic symmetries are essential to induce substantial pair binding of holes in the thermodynamic limit. We argue that this type of model reproduces the ARPES and neutron scattering data seen experimentally.

\section{Introduction and Scenario}

Few recent problems in science have generated so many controversial discussions as the problem of high temperature superconductivity since its experimental discovery in 1986. Two fundamental questions are: Is the superconducting state found in high- $T_{c}$ cuprates homogeneous? Is the superfluid density in these materials homogeneous? The standard approach consists in accepting a homogeneous superconducting state of various forms. We assert that this state is in fact "inhomogeneous." In this work we summarize [1] and expand on a new scenario for striped superconductors where the interplay between inhomogeneous superfluid density and phase fluctuations determines the critical temperature.

In their undoped state, cuprates behave as antiferromagnetic (AF) Mott insulators and it is precisely upon doping with holes that these strongly correlated materials become superconductors. Recent experiments seem to indicate that inhomogeneously textured (intrinsically nanoscale) phases characterize the quantum state of high temperature superconductors. This is, probably, not surprising in retrospect since these are complex materials with competing time and length scales arising from different interactions. A relevant and non-trivial question is, however, whether those textures are essential to drive the phase coherent state, i.e., a Meissner phase.

Neutron scattering experiments have proven to be a very useful tool in investigating magnetic and superconducting properties of high- $T_{c}$ cuprate oxides. With improved sample quality and resolution there is reliable evidence for an incommensurate structure in the spin susceptibility. On the other hand, recent angle-resolved photoemission spectroscopy (ARPES) data suggest a onedimensional (1D) like electronic structure consistent with clustering of charge carriers into 1D channels [2]. Therefore, although the orientation, width, length and dynamics of the channels remains to be elucidated, both the above classes of experiments appear to confirm a new paradigm of spin and charge ordering in high- $T_{c}$ superconductors: the "stripe" phase.

Motivated by this new paradigm we introduced [1] a class of inhomogeneous microscopic models which capture the magnetic and superconducting properties of these strongly correlated materials. The origin(s) of the mesoscopic skeleton of stripe segments in the $\mathrm{CuO}_{2}$ planes is presently unclear and several mechanisms could be responsible, such as local spin-orbit coupling, Jahn-Teller distortions, oxygen buckling at the stripe, and/or other magnetoelastic effects. (Local charge-lattice coupling may be an important source of texture formation.) We showed, in particular, that appropriate inhomogeneous interac- 
tions that break magnetic symmetries are distinctive in inducing substantial pair binding of holes, as well as explaining the magnetic neutron scattering properties. Moreover, based upon the phenomenology of our microscopic model we developed a mean-field ("Josephson spaghetti") model which provides a scenario for the macroscopic superconducting state. We also discussed the connection of the resulting inhomogeneityinduced superconductivity to recent experimental evidence for a linear relation between magnetic incommensurability and the superconducting transition temperature, as a function of doping. In a different work [3] we studied the spectral properties of these inhomogeneous models and found, consistent with experiments [2], a flat band and the correct distribution of quasiparticle weights.

In previous work [1, 3] we have assumed static magnetic inhomogeneities. Certainly, the stripe segments in real materials are likely to have an intrinsic dynamics on a characteristic time scale $\tau$. We assume that this time is large enough for attractive forces to produce bound states of two holes. On the other hand, this stripe dynamics will probably restore the $S U(2)$ spin rotation invariance on time scales greater than $\tau$. $S U(2)$ symmetry does not have to be broken statically. In the present manuscript we also discuss an alternative approach where the magnetic inhomogeneities follow the hole, i.e., the stripe phase is dynamically generated by the holes. This model we call the selfconsistent perturbing hole (SPH) model since the hole itself carries the perturbation. The main qualitative difference between both classes of models is the absence of a broken lattice translational symmetry state in the SPH case. Otherwise, the basic phenomenology is qualitatively the same: Holes pair in stripes as a consequence of the existence of an AF background (avoiding a possible global phase separation). The pairing mechanism is kinetic exchange-interaction based and is provided by magnetic inhomogeneities that locally break spinrotational invariance. In the superfluid phase, it is argued that a phase-locked state is generated as a consequence of a coherent Josephson tunneling of the hole pairs between and along stripes 1 .

\section{Microscopic Inhomogeneous Models}

In this Section we will present two classes of inhomogeneous models. The first model was already introduced in Ref. [1], where the basic microscopic scenario starts from a homogeneous $t-J$ Hamiltonian as background

$H_{t-J}=-t \sum_{\langle\mathbf{r}, \overline{\mathbf{r}}\rangle, \sigma} c_{\mathbf{r} \sigma}^{\dagger} c_{\overline{\mathbf{r}} \sigma}+J \sum_{\langle\mathbf{r}, \overline{\mathbf{r}}\rangle}\left(\mathbf{S}_{\mathbf{r}} \cdot \mathbf{S}_{\overline{\mathbf{r}}}-\frac{1}{4} \bar{n}_{\mathbf{r}} \bar{n}_{\overline{\mathbf{r}}}\right)(1)$

but, to mimic the stripe segments, we add inhomogeneous magnetic interactions. These inhomogeneous terms break translational invariance and spin-rotational $S U(2)$ symmetry locally:

$H_{\mathrm{inh}}=\sum_{\langle\alpha, \beta\rangle} \delta J_{z} S_{\alpha}^{z} S_{\beta}^{z}+\frac{\delta J_{\perp}}{2}\left(S_{\alpha}^{+} S_{\beta}^{-}+S_{\alpha}^{-} S_{\beta}^{+}\right)$

with $\delta J_{\perp} \neq \delta J_{z}$, representing the magnetic perturbation of a static local Ising anisotropy, locally lowering spin symmetry (named a $t-J J_{z}$ model). Only a few links (where the stripes are located) have this lowered spin symmetry. This Ising anisotropy is also sufficient to produce a spin gap.

Pair binding of holes in this class of models is substantial [1]. This substantial binding energy is achieved as the energy for two holes falls much faster with $t$ than twice the energy of one hole.

It is interesting to make the following remarks: Suppose that a stripe segment is represented by the $1 \mathrm{D} t-J_{z}$ model

$H=-t \sum_{\alpha, \sigma}\left(c_{\alpha \sigma}^{\dagger} c_{\alpha+1 \sigma}+\right.$ H.c. $)+J_{z} \sum_{\alpha} S_{\alpha}^{z} S_{\alpha+1}^{z}$. (3)

One can show [4] that, within the ground state subspace (for a given number of holes), this Hamiltonian maps into the attractive spinless fermion model

$H=-t \sum_{\alpha}\left(b_{\alpha}^{\dagger} b_{\alpha+1}+\right.$ H.c. $)-\frac{J_{z}}{4} \sum_{\alpha} \tilde{n}_{\alpha}, \tilde{n}_{\alpha+1}$

where $\tilde{n}_{\alpha}=b_{\alpha}^{\dagger} b_{\alpha}$, and which certainly has a superconducting phase (i.e., correlation exponent $\left.K_{\rho}>1\right)$ [5. In this particular model, it turns out that (e.g., at half-filling for $\left|J_{z} / 8 t\right|<1$ ) the isolated stripe segment belongs to the Luttinger liquid universality class. Notice, however, that 
our stripes are embedded in an AF background. This background provides a strong boundary condition that results in an additional attractive potential for the holes in the stripe. As a result, an enhanced superconducting region is expected [ [], avoiding alternative charge density wave phases or phase segregation.

Knowing that perturbating the system by breaking magnetic symmetries is an efficient mechanism to achieve substantial binding of carriers, some natural questions arise: What would happen if the hole itself carries this perturbation? Would this process be sufficient to generate a stripe phase? Would the binding energy be still appreciable in the thermodynamic limit?

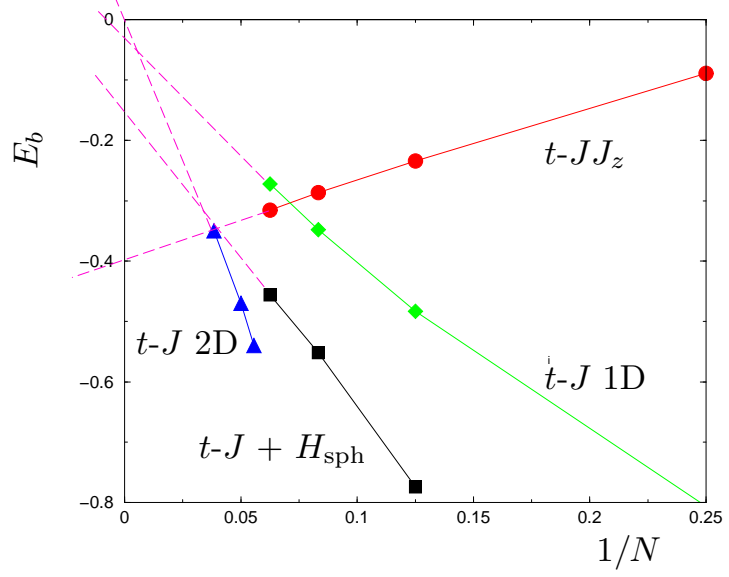

Figure 1. Binding energy $E_{b}$ as a function of $1 / N$ for different models. The lines are guides to the eye. Both homogeneous $t-J$ models in 1D (diamonds) and 2D (triangles) $(t=J=1)$ extrapolate to zero or negligible binding energy. $t-J J_{z}$ extrapolates to a huge binding energy while $t-J+$ $H_{\text {sph }}$ (in both $\delta J_{\perp}=-0.9$ ) extrapolates to moderate binding energy.

Our SPH model corresponds to the Hamiltonian $H=H_{t-J}+H_{\mathrm{sph}}$, where

$$
H_{\mathrm{sph}}=\sum_{\mathbf{r}, \mathbf{d}} \frac{\delta J_{\perp}}{2}\left(1-\bar{n}_{\mathbf{r}}\right)\left(S_{\mathbf{r}}^{+} S_{\mathbf{r}+\mathbf{d}}^{-}+S_{\mathbf{r}}^{-} S_{\mathbf{r}+\mathbf{d}}^{+}\right) .
$$

In this Hamiltonian $1-\bar{n}_{\mathbf{r}}=n_{\mathbf{r}}$ is the ocupation number of holes at site $\mathbf{r}$ and $S_{\mathbf{r}}^{+}, S_{\mathbf{r}}^{-}$are the usual spin operators [1]. The presence of a hole at site $\mathbf{r}$ perturbs the magnetic links in the directions defined by $\mathbf{d}$ by lowering the spin symmetry and making them more Ising-like.

This model is perhaps more natural on physical grounds than the $t-J J_{z}$ one. Magnetoelastic effects caused by the presence of the hole, or buckling of the oxygens close to the carrier may easily produce an Ising-like anisotropy. Upon doping with holes it is not obvious what the extra hole does to the environment. Since these are strongly correlated materials, the extra holes could have a stronger influence on the system than just those effects produced by simple hopping dynamics.

The models considered above are different in some respects. While in the model of Eqs. 1.2 translational symmetry has been explicitly broken (i.e., adding $H_{\mathrm{inh}}$ ) the model defined by $H_{\mathrm{sph}}$ is translationally symmetric, and the only symmetry that has been explicitly broken is the spin $S U(2)$ symmetry around each hole. This fact has some direct experimental consequences. As already discussed in Ref. [1], the $t-J J_{z}$ model has an inhomogeneous hole density, as they prefer to occupy sites where the magnetic links have been weakened (the stripes). This is not the case for the SPH model since, as the translational symmetry has not been broken, the holes will be found with equal probability on every lattice site. This fundamental difference could be resolved by a Scanning Tunneling Microscope experiment.

In this work we will not address the issue of stripe formation in the SPH model, but rather concentrate on the hole pairing in those textures. Clearly there will be a competition between kinetic and magnetic energies. While the first will try to delocalize the pair, the former will contribute to the pairing. In Fig. 1 we show the binding energy (defined as $E_{b}=E_{2}+E_{0}-2 E_{1}$, where $E_{i}$ is the ground state energy in the subspace with $i$ holes) as a function of $1 / N$ (where $N$ is the size of the system). $t-J$ models in $1 \mathrm{D}$ (diamonds) and 2D (triangles) have zero or negligible binding in the extrapolated thermodynamic limit. The model labeled as $t-J J_{z}$ corresponding to the Hamiltonian of Eqs. 1, 2 in 1D has substantial 
binding in the thermodynamic limit. That model
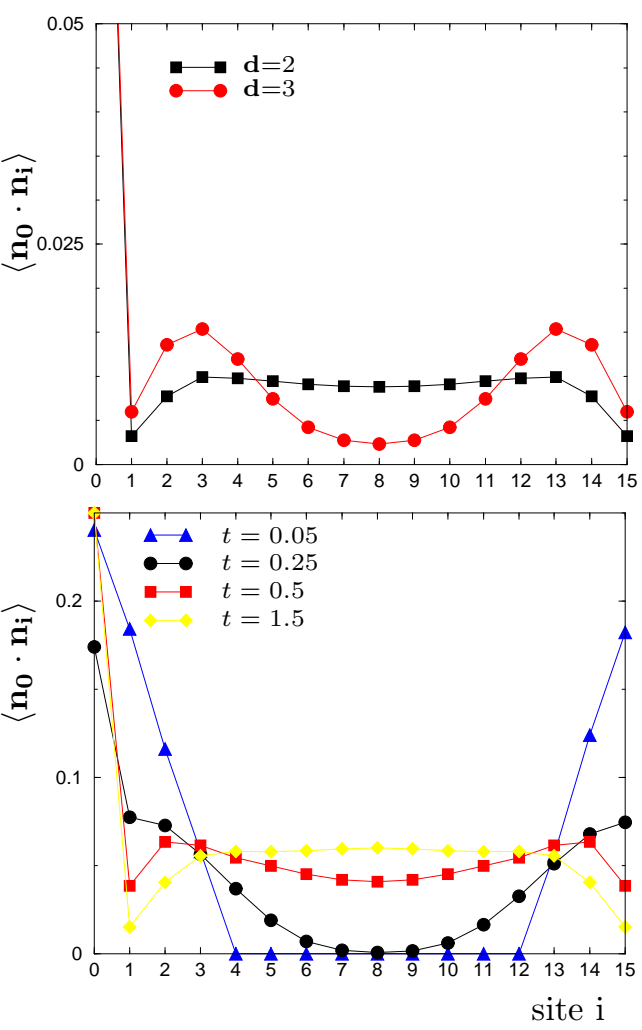

Figure 2. The correlation function $\left\langle n_{0} \cdot n_{i}\right\rangle$, which reflects the shape of the pair, for different cases. Upper panel shows the cases where the perturbation carried by the hole extends to $d=2$ or 3 . Lower panel shows the same correlation function for different values of the kinetic energy $t$. The pair evolves from a tightly bound state to an extended one as the result of a competition between the kinetic energy and the magnetism.

corresponds to placing a perturbation like Eq. 2 every 4 sites. The model labeled as $t-J+H_{\text {sph }}$ is the one we are mostly interested in this work. It has appreciable binding of holes in the thermodynamic limit, although not as strong as in the $t-J J_{z}$ model. In the calculations we have taken $|\mathbf{d}|=2$ (i.e., the hole perturbs up to second neighbors). For $|\mathbf{d}|=1$ the model reduces trivially to the $t-J$ model, as the links close to the holes are magnetically inactive. Therefore $|\mathbf{d}|=d$ must be greater or equal to 2 to show any new behavior. In order to get information about the bound state we have calculated the correlation function $\left\langle n_{0} \cdot n_{i}\right\rangle$, which gives the probability of finding a hole at site $i$ if there is one at site 0 . We remark again that the density of holes is constant and equal to $\left\langle n_{i}\right\rangle=N_{h} / N$ (with $N_{h}$ the number of holes and $N$ the size of the system) for every site $i$ in the case of $t-J+H_{\mathrm{sph}}$. In Fig. 2, upper panel we show this correlation function for the cases $d=2$ and $d=3$ when there are two holes in a 16 sites chain. As expected, the pair is more strongly bound for $d=3$. In a real material there must be a decreasing function of the perturbation carried by the hole with distance. In our case that perturbation is constant. In Fig. 2 lower panel, we show the same correlation function for $d=2$ and different $t$ values. The pair evolves from being tightly bound to delocalization as the kinetic energy is increased. It is worth noting that even when the holes are not very close the binding energy scales to a finite value.

We are grateful to C.D. Batista for useful discussions. This work has been supported by the US DOE.

\section{REFERENCES}

1. J. Eroles, G. Ortiz, A.V. Balatsky, and A.R. Bishop, Europhys. Lett. (in press).

2. X.J. Zhou et al., Science 286, (1999) 268.

3. J. Eroles, G. Ortiz, A.V. Balatsky, and A.R. Bishop (unpublished).

4. C. Batista and G. Ortiz, cond-mat/0003158.

5. A.A. Aligia and G. Ortiz, Phys. Rev. Lett. 82, (1999) 2560. 